\title{
Nuclear Reaction Rates for Astrophysical Nucleosynthesis
}

\author{
A. Palumbo* \\ Department of Physics, Central Michigan University, Mt. Pleasant, MI 48859, USA \\ E-mail: palumla@cmich.edu \\ D. Galaviz \\ Centro de Fisica Nuclear da Universidade de Lisboa, 1649-003, Lisbon, Portugal \\ E-mail: galaviz@ciencias.ulisboa.pt
}

\begin{abstract}
A new reaction rate formalism is under development and preliminary results are presented here. Particular emphasis is placed on the astrophysical relevance for heavy element synthesis. Charged particle, neutron induced, and photonuclear reactions of heavy nuclei formed in the r-, s- and pprocesses show an overall consistent agreement. An estimation of the error one can anticipate from the calculated reaction rates is included. The reaction rates within the astrophysical regime and in comparison to experimental reaction rates from data and using different nuclear inputs illustrate the dominating HF component. Results in comparison to established model codes are shown and very good agreement is met. This illustrates the viability of such an implementation for abundance studies. Once the formalism is fully implemented, we anticipate a powerful available tool for use in calculations of astrophysical relevance.
\end{abstract}

XIII Nuclei in the Cosmos,

7-11 July, 2014

Debrecen, Hungary

${ }^{*}$ Speaker. 


\section{Introduction}

The majority of heavy elements beyond the Fe/Ni peak are synthesized via neutron captures in the r-process and s-process. Completing the synthesis beyond this point are photodissociations in a hot photon bath (the $\gamma$-process [1], [2]), a series of rapid proton captures (rp-process [3]) and a strong neutrino flux process for the light p-nuclides ( $v$-process $[4,5])$ via which all of these processes contribute to the formation of the p-nuclei. The bulk of nuclei formed in all of these processes assume a high enough level density such that the Hauser Feshbach (HF) formalism holds [6].

Since simulations for processes such as the $r$ - and p- involve an extensive network of many reactions where data is as of yet currently unavailable (most of the reactions are on radioactive, short-lived nuclei), these rates are theoretically based and extrapolations using nuclear models can be problematic.

The reaction rates presented here are currently based on the formalism from TALYS [7]. The cross sections are taken from the EMPIRE code [8] which takes its nuclear inputs from the RIPL database [9]. The errors for the reaction rates are calculated as a sum in quadrature of the dominating nuclear physics ingredients of the cross sections (and in arriving at an error estimate for the calculated cross sections, several approximations of the HF formalism were taken). These components include the nuclear level density (NLD), the gamma strength function $(\gamma \mathrm{sf})$, the optical model potential (OMP) and the width fluctuation coefficient (WFC). Table 1 shows some of the dominant Hauser Feshbach (HF) ingredients that go into a nuclear reaction rate. The particular combinations (Enhanced Generalized Superfluid model (EGSM), Modified Lorentzian (MLO1) and enhanced generalized Lorentzian (EGLO)) which are standard in EMPIRE and used in the nuclear astrophysics community are shown with their corresponding error and were used in the nuclear reaction rates presented here.

\begin{tabular}{llllll}
\hline Reaction & OMP & NLD & $\gamma$-strength & Error (\%) & COMBO \\
\hline$(\alpha, \gamma)$ & McFadden/Satchler [11] & EGSM [12, 13] & MLO 1 [16] & 26 & 1 \\
$(\alpha, \gamma)$ & Avrigeanu [10] & EGSM [12, 13] & MLO 1 [16] & 29 & 2 \\
$(\mathrm{p}, \gamma)$ & Koning-Delaroche [18] & EGSM [12, 13] & MLO 1 [16] & 23 & 1 \\
$(\mathrm{p}, \gamma)$ & Koning-Delaroche [18] & EGSM [12, 13] & EGLO [17] & 20 & 2 \\
$(\mathrm{p}, \gamma)$ & Koning-Delaroche [18] & HFB [14, 15] & MLO 1 [16] & 22 & 3 \\
$(\mathrm{p}, \gamma)$ & Koning-Delaroche [18] & HFB [14, 15] & EGLO [17] & 20 & 4 \\
$(\mathrm{n}, \gamma)$ & Koning-Delaroche [18] & EGSM [12, 13] & MLO 1[16] & 25 & 1 \\
$(\mathrm{n}, \gamma)$ & Souhovitski [30] & EGSM [12, 13] & MLO 1[16] & 25 & 2 \\
\hline
\end{tabular}

Table 1: Comparison of the different nuclear inputs and their corresponding error for the reaction rates from EMPIRE [8]. Combo refers to the HF combination used in Fig.1

\section{Charged particle reactions}

The bulk of the p-nuclei are formed in a hot $(\mathrm{T} \approx 1.8-3.2 \mathrm{GK})$ photon bath starting with a pre-existing seed whereby photodissociations are the main method of synthesis. Charged particle 
reactions are hindered by the Coulomb barrier and measurements within the astrophysically relevant Gamow window are difficult to obtain due to the low cross section. Problems with agreement to the Hauser Feshbach (HF) statistical model have mainly arisen due to the $\alpha$-potential in the case of $\alpha$-induced measurements; however, the $\gamma$-width could also cause deviations. This is particularly true below the neutron threshold, where other HF components may be causing a problem (neutron widths are broad and normally there are not many isolated resonant components above the threshold where many neutrons are being emitted) [19]. Although the agreement to proton capture has overall been better in comparison to the theory, it is difficult to individually pinpoint the sensitivity of the optical potential, level density and the $\gamma$-strength function.

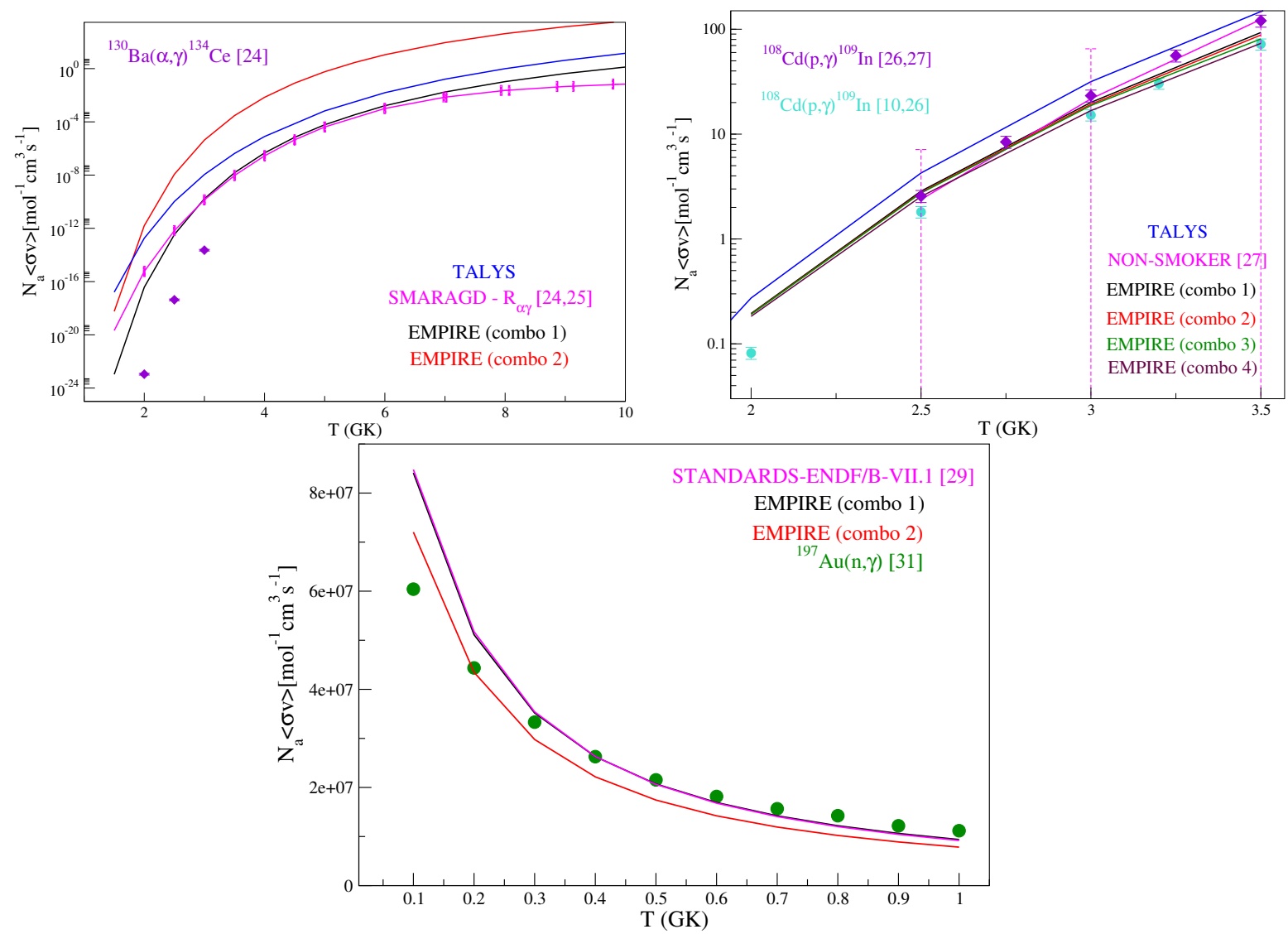

Figure 1: $\alpha$-capture on ${ }^{130} \mathrm{Ba}$ and $\mathrm{p}$-capture on ${ }^{108} \mathrm{Cd}$ and $\mathrm{n}$-capture on ${ }^{197} \mathrm{Au}$ in comparison to the $\mathrm{HF}$ model predictions. The SMARAGD value have been assigned an uncertainty of 3 while the NON-SMOKER values have been assigned an uncertainty of 2 in the graph.

Fig. 1 shows ${ }^{130} \mathrm{Ba}(\alpha, \gamma){ }^{134} \mathrm{Ce}[20]$ in comparison to the HF model predictions. The default values for ${ }^{130} \mathrm{Ba}(\alpha, \gamma){ }^{134} \mathrm{Ce}$ of TALYS [7] match the values from EMPIRE [8] using Combo 2 up to $\mathrm{T}_{9}=2 \mathrm{GK}$. The Gamow window for the ${ }^{130} \mathrm{Ba}(\alpha, \gamma){ }^{134} \mathrm{Ce}$ reaction falls between 5.3 to $8.1 \mathrm{MeV}$ for $\mathrm{T}_{9}=2 \mathrm{GK}$. Combo 2 uses the Avrigeanu potential, EGSM level density and MLO1 $\gamma$-strength function. The measured energy points at $11.6-16 \mathrm{MeV}$ (both ${ }^{130} \mathrm{Ba}(\alpha, \mathrm{n})$ and ${ }^{130} \mathrm{Ba}(\alpha, \mathrm{p})$ are open in this energy range) were input into the reaction rate calculation for temperatures of 2, 2.5 and $3 \mathrm{GK}$. The errors for the reaction rates for the data were determined using the experimentally quoted values. A visual inspection in the optimization of the calculations of the code to the data 
showed that the dominating HF component was the $\alpha$-potential. This is also confirmed by the fact that SMARAGD [21] and EMPIRE (combo 1) use the same $\alpha$-optical potential while TALYS and EMPIRE (combo 2) use different $\alpha$-optical potentials. Combo 1 is the McFadden/Satchler potential and Combo 2 is the Avrigeanu potential. Both combinations from EMPIRE use the MLO1 $\gamma$-strength function and the EGSM level density.

\begin{tabular}{llll}
\hline $\mathrm{T}(\mathrm{GK})$ & Rate (EMPIRE) & $R_{\alpha \gamma}(\mathrm{SMARAGD})$ & $\%$ diff \\
\hline 2 & $3.70( \pm 0.85) \times 10^{-17}$ & $5.827 \times 10^{-16}$ & 20.4 \\
2.5 & $3.45( \pm 0.794) \times 10^{-13}$ & $6.798 \times 10^{-13}$ & 1.6 \\
3 & $1.69( \pm 0.389) \times 10^{-10}$ & $1.378 \times 10^{-10}$ & 1.1 \\
\hline
\end{tabular}

Table 2: Comparison of EMPIRE and SMARAGD reaction rates for ${ }^{130} \mathrm{Ba}(\alpha, \gamma){ }^{134} \mathrm{Ce}$

Table 2 shows the reactivities from SMARAGD [20] and the reaction rates from EMPIRE (COMBO 1) for ${ }^{130} \mathrm{Ba}(\alpha, \gamma){ }^{134} \mathrm{Ce}$. The values for the reaction rates at 2.5 and $3 \mathrm{GK}$ from EMPIRE and SMARAGD fall within the errors given by the values from EMPIRE; however, the value at 2 GK deviates outside of the error range. Both use the same $\alpha$-potential but differing level density and $\gamma$-strength functions. This suggests that either the $\alpha$-optical potential is the dominating problematic component or that there may be some other contributing HF component in this low energy regime $(\mathrm{T}=2 \mathrm{GK})$.

The ${ }^{108} \mathrm{Cd}(\mathrm{p}, \gamma){ }^{109}$ In was optimized using the EGSM NLD and the EGLO $\gamma$-strength function. For proton captures, it is difficult to distinguish the dominating sensitivity of the NLD, the $\gamma$ strength function and the optical potential. Table 3 shows the values of the reaction rates from EMPIRE using the EGSM level density and the EGLO $\gamma$-strength function with the theoretical values from [22]. Also shown are the experimental reaction rates obtained using the cross sections from [22] and input into the reaction rate formalism from EMPIRE and also obtained using the code EXP2RATE [22].

\begin{tabular}{lllll}
\hline $\mathrm{T}(\mathrm{GK})$ & Rate (EMPIRE) & Rate [22, 23] & Rate (EMPIRE [22]) & Rate [22] \\
\hline 2.5 & $2.79 \pm 0.56$ & 2.38 & $1.81 \pm 0.227$ & $2.56 \pm 0.34$ \\
3 & $19.25 \pm 3.85$ & 21.7 & $15.2 \pm 1.86$ & $23.3 \pm 3.04$ \\
\hline
\end{tabular}

Table 3: Comparison of EMPIRE and NONSMOKER for ${ }^{108} \mathrm{Cd}(\mathrm{p}, \gamma)$. The fourth column are the values from [22] fed into the reaction rate formalism from EMPIRE.

The theoretical calculation of the reaction rate for ${ }^{108} \mathrm{Cd}(\mathrm{p}, \gamma){ }^{109}$ In using EMPIRE and the values from [22] agree within the errors for all combinations above $\mathrm{T}_{9}=2.5 \mathrm{~K}$; however, the values obtained from the experimental cross section data using the reaction rate formalism from EMPIRE and the reaction rates from the code EXP2RATE [22] using the experimental data [22] do not agree within the errors. The experimental reaction rates obtained from [22] considers the possible temperature window whereas the experimental values from [22] were input into the EMPIRE reaction rate calculation and all available measured energies were considered in the calculation. One sees a 
marked deviation at $\mathrm{T}=3 \mathrm{GK}$. It is also interesting to note that although a different level density, optical potential and $\gamma$-strength function were used between the two theory model codes, there is overlapping agreement for the theoretically derived reaction rates. Although the measurement was obtained below the opening of the neutron threshold, where in principle only the $p, \gamma$ channel is open (the neutron threshold opens at $6 \mathrm{MeV}$ ), there is no conclusion on which HF component is more sensitive. Recent measurements of the Cd isotopes [24] have revealed the presence of some extra low lying strength (or pygmy dipole resonance) in ${ }^{106} \mathrm{Cd}$ with inconclusive results for ${ }^{108} \mathrm{Cd}$ due to lack of available data [24]. The general trend is an increasing pygmy strength as the neutron number increases [24]. This extra strength is of main interest for the reaction rates of the r-process (close to the driplines) but this strength needs to be addressed by the correct representation of the $\gamma$-strength function model.

TALYS and EMPIRE both use the Koning Delaroche optical potential and therefore this is excluded as a problematic HF component in this case; therefore, the deviation could possibly be between the level density and the $\gamma$-strength function as the difference arising between the three model codes: EMPIRE, NON-SMOKER [23] and TALYS. All three codes use a different level density; therefore, the difference could be due to the $\gamma$-strength function (the version of the enhanced generalized lorentzian $\gamma$-strength function is different from TALYS to EMPIRE).

\section{Neutron capture reactions}

Neutron capture cross sections in the mass region of the weak s-process are not often available with the required accuracy for abundance determination. Although not of direct astrophysical relevance, the nuclear astrophysics community has used the neutron capture cross section of ${ }^{197} \mathrm{Au}(\mathrm{n}, \gamma)$ from Ratynski and Käppeler [25] as the standard for the neutron flux normalization in neutron capture measurements. This result was used to normalize the ${ }^{197} \mathrm{Au}(\mathrm{n}, \gamma)$ cross section of Macklin to obtain a reference cross section [26]. The nuclear data community considers the ${ }^{197} \mathrm{Au}(\mathrm{n}, \gamma)$ cross section as a standard from $200 \mathrm{keV}$ to $2.5 \mathrm{MeV}$ based on the evaluation by Carlson [27]. A new measurement [28] reports a value for the MACS (at kT $=30$ ) of $626 \pm 25 \mathrm{mb}$.

A new $n \_$TOF measurement has obtained the neutron capture cross section in the energy range from 5 to $400 \mathrm{keV}$ [29]. Fig. 1 shows the reaction rate of ${ }^{197} \mathrm{Au}$ using the cross sections obtained via n_TOF [29] where the cross section values used as input for the reaction rate have been obtained by energy averaging between bins. Fig. 1 also shows the reaction rate obtained from the data in comparison to the standards [27], the default calculation by EMPIRE (combo 1) and an EMPIRE calculation using the potential by Souhovitski (combo 2). The rate is that of the groundstate. Combo 1 and the calculation with the Souhovitski potential [30] both use the EGSM level density and the EGLO $\gamma$-strength function. Outside of the range of the standards [27], the lowest two data points agree within the error of the Souhovitski potential while the higher data points agree within the errors. If one assumes the same error for the Standards, then one sees that it is in agreement with the TOF data (which introduces an approximate error of 3.9\% - 6.7\%).

\section{Conclusion}

Reaction rates of relevance to heavy element synthesis in the $\gamma$-, and s- processes have been 
presented here. The rates (together with the tabulated errors from the EMPIRE code) show good agreement not only to the data but also to other statistical model codes. This reaction rate formalism is in its preliminary stages but once implemented, it should be a powerful tool for reaction rates of interest to heavy element synthesis.

\section{References}

[1] T. Rauscher, N. Dauphas, et al., Rep. Prog. Phys. 76, (2013) 066201.

[2] M. Arnould and S. Goriely, Physics Reports 384, (2003) 1-84.

[3] H. Schatz, et al., Physics Reports 294, (1998) 167-263.

[4] C. Fröhlich, et al., PRL 96, (2006) 142502.

[5] A. Arcones, C. Fröhlich, and G. Martinez-Pinedo, The Astrophysical Journal 750, (2012) May 1.

[6] W. Hauser, H. Feshbach, Phys. Rev. 87 (1952) 366.

[7] A.J. Koning and D. Rochman, Modern nuclear data evaluation with the TALYS code system, Nucl. Data Sheets 113, 2841 (2012).

[8] M. Herman, R. Capote et al., Nucl. Data Sheets 108, (2007) 2655-2715.

[9] R. Capote et al., Nuclear Data Sheets 110 Issue 12 (2009) 3107-3214.

[10] M. Avrigeanu et al., ADNDT 95 (2009) 501-532.

[11] L. McFadden and G.R. Satchler, Nucl. Phys. A 84 (1966) 177.

[12] A. D'Arrigo et al., Phys. Rev. C 46 (1992) 1437.

[13] A. D’Arrigo et al., Phys. G: Nucl. Part. Phys. 20 (1994) 365-376.

[14] S. Goriely et al., Phys. Rev. C 75, (2007) 064312.

[15] S. Goriely et al., Phys. Rev. C 78, (2008) 064307.

[16] V.A. Plujko, Acta Phys. Pol. B, 31, (2000) 435.

[17] J. Kopecky, M. Uhl, and R.E. Chrien, Phys. Rev. C, 47, (1993) 312.

[18] A.J. Koning and J.P. Delaroche, Nucl. Phys. A 713, (2003) 231-310.

[19] A. Palumbo, Proceedings of Science, Nuclear in Physics in Astrophysics VI (2013).

[20] Z. Halász, et al., Physical Review C 85, (2012) 025804.

[21] Rauscher T 2011 The path to improved reaction rates for astrophysics Int. J. Mod. Phys. E 201071.

[22] Gy. Gyürky, et al., J. Phys. G: Nucl. Part. Phys. 34, (2007) 817-825.

[23] Rauscher T and Thielemann FK, At. Data and Nucl. Data Tables 79 (2001) 47.

[24] A.C. Larsen et al., Phys. Rev. C 87 (2013) 014319.

[25] W. Ratynski, F. Käppeler, Phys. Rev. C 37 (1988) 595.

[26] R.L. Macklin and R.H. Gibbons, Phys. Rev. C159 (1967) 1007.

[27] A.D. Carlson et al., Nucl. Data Sheets 110 (2009) 3215.

[28] P. Jimenez-Bonilla and Javier Praena, Proceedings of Science, XIII Nuclei in the Cosmos, (2014).

[29] C. Lederer, Phys. Rev. C 83 (2011) 034608.

[30] O. Bersillon, CEA Report CEA-N-2284 (1982) p.130 and NEANDC-222U. 